\title{
Adaptación transcultural colaborativa de cuestionarios PCAT para Colombia
}

\author{
Collaborative transcultural adaptation of the PCAT questionnaires for Colombia
}

Adaptação transcultural colaborativa de questionários PCAT para Colômbia

\author{
Alexandra Giraldo-Osorio* \\ Consuelo Vélez-Álvarez** \\ M Isabel Pasarin ${ }^{* * *}$ \\ Jacqueline Ponzo**** \\ Silvina Berra ${ }^{* * * *}$
}

\section{Resumen}

Objetivo: Obtener versiones de los cuestionarios Primary Care Assessment Tool (PCAT) para Colombia (CO-PCAT), dirigidos a profesionales (PCAT-PE), gestores y directivos de atención primaria (PCAT-FE) a través de un proceso colaborativo internacional. Materiales y Métodos: Proceso de adaptación transcultural modificado a partir de las versiones adaptadas y validadas en español, en consenso con la Colaboración Iberoamericana IA-PCAT. El método busca conseguir la equivalencia de la versión CO-PCAT de los cuestionarios con la versión original de Estados Unidos de América. Se desarrollaron tres fases de trabajo: I) valoración de la adecuación de versiones iberoamericanas en lengua española y adaptación al contexto colombiano, II) revisión internacional realizada por investigadores de la Colaboración Iberoamericana PCAT y III) pruebas preliminares de los cuestionarios. Consideraciones éticas: es una investigación sin riesgos para los seres humanos conforme a la Resolución 08430 de 1993 de Colombia y la Declaración de Helsinki. Resultados: Del PCAT-PE se incluyeron en la versión colombiana 182 ítems. En cuanto al PCAT-FE se trabajó con 197 ítems en total. Conclusión: Se obtuvieron versiones adaptadas al sistema de salud colombiano. La metodología empleada puede contribuir al logro de mayor equivalencia entre instrumentos de diferentes países.

Palabras clave: atención primaria de salud, servicios de salud, encuestas y cuestionarios, Colombia.

\footnotetext{
Abstract

Objective: Obtain versions of the Primary Care Assessment Tool (PCAT) questionnaires for Colombia (CO-PCAT), directed to professionals (PCAT-PE), managers and directives of primary care (PCAT-FE) through an international, collaborative process. Materials and Methods: A transcultural adaptation process modified from the adapted and validated Spanish versions, in consensus with the Ibero-American Collaboration IA-PCAT. The method intends to get an equivalence of the CO-PCAT questionnaires
}

Autor de correspondencia

* Enfermera. Magíster en Salud Pública. Investigadora del Grupo Promoción de la Salud. Docente Universidad de Caldas. Correo: alexandra.giraldo@ucaldas.edu.co. (iD https://orcid. org/0000-0003-1330-2713. Manizales, Colombia.

** Enfermera. Doctora en Salud Pública. Investigadora Grupo de Investigación en Promoción de la Salud y Prevención de la Enfermedad. Docente Universidad de Caldas. Correo: consuelo.velez@ucaldas.edu.co. (iD) https://orcid. org/0000-0001-7274-7304. Manizales, Colombia.

*** Médica. Doctora en Salud Pública y Metodología de la Investigación Biomédica. Agència de Salut Pública de Barcelona. IIB Sant Pau. CIBER de Epidemiología y Salud Pública. UPF. España.. Correo: mpasarin@aspb.cat. (iD https://orcid.org/0000-0002-4809-1250. Barcelona, España.

${ }^{* * * *}$ Médica. Especialista en Medicina Familiar y Comunitaria, Magíster en Epidemiología. Investigadora Grupo PCAT.UY, Universidad de la República. Correo: ponzo4@gmail.com. (iD) https://orcid.org/0000-0002-8329-7430. Canelones al Este, Uruguay.

***** Nutricionista. Doctora en Ciencias de la Salud. Centro de Estudios e Investigaciones Sobre Cultura y Sociedad (CIECS) CONICET; Escuela de Salud Pública, Facultad de Ciencias Médicas. Correo: sberra@fcm.unc.edu.ar. (ID https://orcid.org/0000-0003-3618-7041. Córdoba, Argentina.

Este es un artículo bajo la licencia CC BY

(https://creativecommons.org/

licenses/by/4.0/) @() (1) 
ISSN-PRINT

1794-9831

E-ISSN 2322-7028

Vol. 15 No. 2

Jul - Dic 2018

Cúcuta, Colombia

version with the original version of the United States. Three phases of the work were developed: I) assessment of the adaptation of ibero-american versions in Spanish and adaptation to the Colombian context. II) international review performed by researchers of the Ibero-American Collaboration PCAT, and III) preliminary trials of the questionnaires. Ethical considerations: it is a non-risk research for human beings according to the Resolution 08430 of 1993 of Colombia and the Declaration of Helsinki. Results: From the PCAT-PE, 182 items were included in the Colombian version. Regarding PCATPE, the research worked with a total of 197 items. Conclusion: Adapted versions to the Colombian health system were obtained. The methodology used can contribute to the accomplishment of a greater equivalence between instruments of different countries.

Keywords: primary health care, health services, surveys and questionnaires, Colombia.

\section{Resumo}

Objetivo: Obter versões dos questionários Primary Care Assessment Tool (PCAT) para Colômbia (CO-PCAT), dirigidos a professionais (PCAT-PE), encargados e diretivos de atenção primária (PCATFE) através de um processo colaborativo internacional. Materiais e Métodos: Processo de adaptação transcultural modificado a partir das versões adaptadas e validadas em espanhol, em consenso com a Colaboração Ibero-americana IA-PCAT. O método procura alcançar a equivalência da versão CO-PCAT dos questionários com a versão original de Estados Unidos da América. Desenvolveram-se três fases de trabalho: I) valoração da adequação de versões Ibero-americanas em língua espanhola e adaptação ao contexto colombiano, II) revisão internacional realizada por pesquisadores da Colaboração Iberoamericana PCAT e III) testes preliminares dos questionários. Considerações éticas: É uma pesquisa sem riscos para os seres humanos conforme à Resolução 08430 de 1993 da Colômbia e a Declaração de Helsinki. Resultados: do PCAT-PE se incluíram na versão colombiana 182 itens. Em quanto ao PCATFE se trabalho com 197 itens em total. Conclusão: Obtiveram-se versões adaptadas ao sistema de saúde colombiano. A metodologia utilizada pode contribuir para a obtenção de maior equivalência entre instrumentos de diferentes países.

Palavras-chave: Atenção primaria de saúde, serviços de saúde, pesquisas e questionários, Colômbia.

\section{Introducción}

Según Sperber (1), la adaptación transcultural se ha realizado principalmente en el área de ciencias sociales y posteriormente en salud, reconociendo su importancia, particularmente en el uso de instrumentos para evaluar estrategias como la atención primaria de salud (APS). Los investigadores que necesitan instrumentos adecuados para medir el grado en que la APS cumple sus funciones primarias y secundarias, tienen dos opciones: desarrollar un nuevo instrumento o modificar un instrumento previamente validado en otro idioma (2). En el segundo caso se requiere un proceso de adaptación transcultural, según lo planteado por Shi et al. (3). Acorde con lo descrito por Vélez y Giraldo (4), la APS ha sido reconocida como una estrategia válida para hacer frente a los desafíos que los sistemas de salud enfrentan, debido a que incluye todos los grupos poblacionales y tiene en cuenta el desarrollo de acciones de promoción de la salud, prevención de la enfermedad, protección específica y detección temprana, entre otras razones.
Diferentes gobiernos han realizado reformas en los sistemas de salud en países de América Latina y el Caribe basados en la APS. De acuerdo con Shi et al. (3), en este contexto es deseable que estos procesos cuenten con mecanismos de evaluación que permitan establecer en qué medida dicha estrategia se está ejecutando, su calidad, eficiencia, costo, equidad y satisfacción de los usuarios. Starfield et al. (5), autores del Primary Care Assessment Tool ( PCAT por su sigla en inglés), lo definen como un conjunto de herramientas disponibles para evaluar el grado en que los servicios de salud del primer nivel de atención cumplen con los atributos esenciales y secundarios de la APS (primer contacto, longitudionalidad, coordinación e integralidad; orientación comunitaria, enfoque familiar y competencia cultural); herramientas de las que deben dispone los profesionales del equipo de salud, los gestores o directivos y los usuarios. Estos cuestinarios de evaluación fueron desarrollados en la Universidad John Hopkins de los Estados Unidos de América y han sido adaptados para su aplicación en diferentes países de Iberoamérica -Argentina 
(6), Brasil (7-10), Uruguay (11), España (12-14), Ecuador- así como en países de otros continentes China (15), Corea (16) y Sudáfrica-.

\section{Objetivos}

\section{Objetivo general}

Obtener versiones de los cuestionarios PCAT para Colombia dirigidos a profesionales (CO-PCATPE), gestores o directivos (CO-PCAT-FE) de APS; con equivalencia semántica, práctica y cultural a las versiones originales y su adecuación al sistema de salud colombiano, a través de un proceso colaborativo internacional.

\section{Objetivos específicos}

- Traducir las versiones extensas de los cuestionarios PCAT-PE y PCAT-FE.

- Evaluar la adecuación al sistema de salud colombiano de las versiones CO-PCAT-PE y CO-PCAT-FE vigilando su validez de contenido.

- Valorar la comprensión y equivalencia semántica del contenido de las versiones adaptadas por parte de personas de la población objetivo.

\section{Materiales y Métodos}

La Colaboración IA-PCAT propuso una modificación de la metodología de adaptación transcultural que permitiera capitalizar la experiencia acumulada en los diferentes procesos nacionales de adaptación a países de la región, estudio desarrollado entre 2008 y 2012, según las publicaciones reportadas en su portal Web (17). En este caso, se planteó iniciar el proceso de adaptación transcultural partiendo de las versiones en español ya existentes en la región, manteniendo como referencia la versión original de Estados Unidos de América y la versión de Brasil. El método se propone encontrar la equivalencia de la versión CO-PCAT de los cuestionarios con la versión original, adaptándola culturalmente al contexto colombiano, con la mayor proximidad posible a las versiones adaptadas y disponibles en países de la región; al respecto Beaton et al. (18) plantean una medición equivalente ante diferencias idiomáticas, relacionadas con el entorno de salud y social de un país respecto a otros, teniendo en cuenta que las palabras y las expresiones significan lo mismo, así como las experiencias de la vida diaria capturadas por el cuestionario que son vividas de forma equivalente entre una cultura y otra. Se desarrollaron tres fases de trabajo (Figura 1).

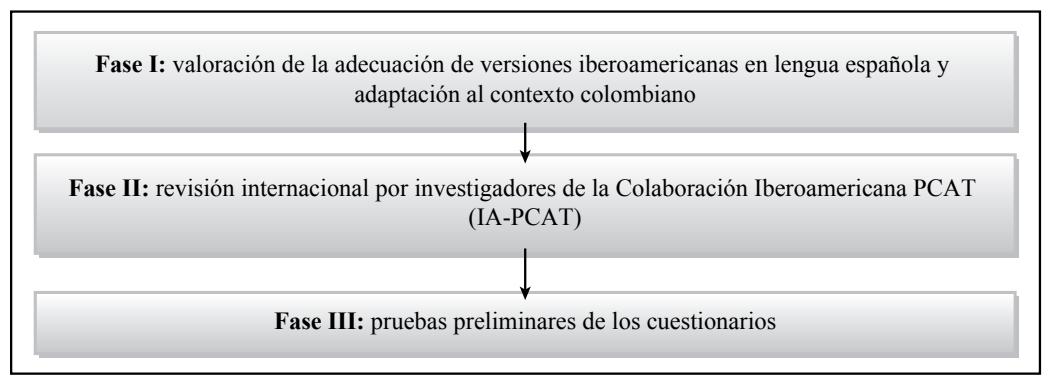

Figura 1. Esquema de la metodología utilizada para la adaptación transcultural de los instrumentosparaprofesionalesygestoresodirectivosdelsistemadesaludcolombiano.

Fuente: elaboración propia.

I. Valoración de la adecuación de versiones iberoamericanas en lengua española y adaptación al contexto colombiano

Se dispuso de las versiones para profesionales (providers edition; PE) de Argentina (AR-PCAT-PE) (6), Uruguay (UR-PCAT-PE) (11) y para gestores o directivos (facility edition; FE) de Argentina (ARPCAT-FE) (6) y España (ES-PCAT-FE) (13). Se diseñó una plantilla que incluyera esas versiones y las de referencia (Estados Unidos de América, US; y
Brasil, BR) con celdas para registrar todas las fases del proceso.

Se conformó un grupo de trabajo de cinco expertos colombianos en APS y las investigadoras locales (Giraldo y Vélez) con el propósito de revisar la adecuación del lenguaje y del contenido de cada uno de los ítems al sistema de salud colombiano (Tabla 1). Para la elección de los participantes se tuvieron en cuenta los siguientes criterios: diferentes disciplinas, diferente actividad y diversos ámbitos o jurisdicciones de desempeño en el 
ISSN-PRINT

1794-9831

E-ISSN 2322-7028

Vol. 15 No. 2

Jul - Dic 2018

Cúcuta, Colombia sistema de salud. Los participantes fueron contactados a partir de referentes locales y nacionales en APS (academia, instituciones prestadoras de servicios de salud y direcciones seccionales de salud municipales y departamentales), para luego ser capacitados en los fundamentos teóricos (funciones de la APS) sobre los que se construyeron los cuestionarios, los objetivos del proceso de adaptación y la metodología necesaria para conseguir la equivalencia semántica, la práctica y la cultural. Se realizó un ejercicio de calibración inicial para la calificación de los ítems.

Los expertos calificaron cada uno de los ítems de acuerdo a los siguientes niveles definidos por la colaboración IA-PCATl: nivel I (ítem equivalente, es decir, podría utilizarse el mismo enunciado), nivel II (requiere cambios mínimos, necesarios para su adaptación), nivel III (requiere cambios mayores necesarios para su adaptación) y nivel IV (ítem no equivalente, debería excluirse). Cuando lo consideraron necesario, hicieron propuestas de modificación del texto de los ítems.

A continuación, se realizó una revisión por parte de las investigadoras colombianas, identificando los ítems con mayor divergencia y controlando la equivalencia semántica, práctica y cultural. Como producto de esta fase se obtuvieron las primeras versiones adaptadas para Colombia de los cuestionarios y se elaboró un informe como insumo para la fase 2.

Tabla 1. Conformación Grupo de Expertos PCAT-PE y PCAT-FE.

\begin{tabular}{lll}
\hline Expertos & Perfil & Ámbito de desempeño \\
\hline No. 1 & $\begin{array}{l}\text { Odontóloga, Especialista en Salud Ocupacional, Magíster en } \\
\text { Salud Pública }\end{array}$ & Institución de educación superior \\
No. 2 & $\begin{array}{l}\text { Médico, Especialista en Administración de Sistemas y Servicio } \\
\text { de Salud, Magíster en Salud Pública }\end{array}$ & $\begin{array}{l}\text { Asesor de Sistemas y Servicios de Salud } \\
\text { de la OPS/OMS }\end{array}$ \\
No. 3 & $\begin{array}{l}\text { Enfermera, Especialista en Promoción de la Salud, Magíster en } \\
\text { Salud Pública }\end{array}$ & $\begin{array}{l}\text { Empresa Social del Estado de primer } \\
\text { nivel de atención en salud }\end{array}$ \\
No. 4 & $\begin{array}{l}\text { Médico, Magíster en Salud Pública, Magíster en Política y } \\
\text { Gestión de Salud, Doctor en Salud Pública }\end{array}$ & Secretaría de Salud Departamental \\
\hline No. 5 & Fisioterapeuta, Especialista en Gerencia en Salud Ocupacional & Secretaría de Salud Municipal \\
\hline
\end{tabular}

Fuente: Elaboración propia.

\section{Revisión internacional por investigadores de la Colaboración Iberoamericana PCAT (IA-PCAT)}

Las investigadoras de Argentina (Berra), Uruguay (Ponzo) y España (Pasarín) revisaron el informe del proceso llevado a cabo en Colombia, controlando que los cambios propuestos lograrán mantener la equivalencia entre las versiones de todos los países y con las originales. Cada revisora trabajó individualmente, a continuación se efectuó una conferencia internacional para discutir las observaciones realizadas hasta lograr acuerdos ítem por ítem. Las responsables del proyecto en Colombia ajustaron las primeras versiones obtenidas en función de los acuerdos alcanzados en esta fase.

\section{Pruebas preliminares de los cuestionarios}

Esta fase se tuvo como propósito el valorar la comprensión de los términos y preguntas, la claridad y aceptabilidad del lenguaje, así como la dificultad práctica para responderlos por parte de personas de la población diana de los instrumentos. Previamente, se elaboró un listado de ítems con base en los resultados obtenidos en las Fases 1 y 2 sobre los que se debía prestar mayor atención durante las pruebas. En la realización de dicho listado se tuvieron en cuenta los ítems que sufrieron modificaciones importantes en las fases previas por haber sido valorados en los niveles II y III; la versión resultante fue socializada con el grupo de expertos nacionales para que evaluaran los niveles de acuerdo frente al proceso adelantado.

Para la prueba de comprensión, se seleccionaron entre 4 y 16 personas del colectivo al que iba dirigido cada uno de los cuestionarios, número considerado suficiente para este tipo de estudios acorde con Guillemin et al. (19).

Los participantes fueron contactados a partir de referentes locales en atención primaria de salud (academia y Secretaría de salud). Los criterios para seleccionarlos fueron: participación en el equipo de salud desde un nivel profesional o técnico en el proceso de atención a la salud (médico, enfermero, psicólogo, trabajador social, agente de salud pública, técnico.... entre otros); con desempeño en 
diferentes ámbitos (nacional, departamental, municipal); desempeño en diferentes roles y sector (público, privado). Para la selección de los gestores y directivos a quienes se les aplicaría el formulario se tuvo en cuenta su desempeño como jefes de centros o de equipos de atención primaria o que tuvieran responsabilidad en la administración de servicios.

Para la aplicación de los cuestionarios se seleccionaron servicios de salud de características diversas, en concordancia con las particularidades del sistema de salud colombiano.

Ambos cuestionarios (CO-PCAT-PE y CO-PCATFE) podían ser respondidos por autoadministración. El entrevistador debía ir haciendo pequeñas marcas en preguntas donde notara alguna dificultad, sin interrumpir la administración. Al finalizar la entrevista, se indagaba sobre aspectos concretos del cuestionario con la utilización de una guía, en donde cada entrevistador registraba exhaustivamente todo lo sucedido, con notas de campo, bien durante la entrevista o al terminarla. Las guías incluían el listado de los ítems a probar antes mencionados, más cuatro preguntas sobre: dificultad, comprensión, interés y adecuación del lenguaje del cuestionario; las respuestas se calificaron de acuerdo con una escala de 5 opciones $(1=$ nada; $2=$ un poco; $3=$ moderadamente; 4=mucho; 5=muchísimo). A continuación se leía cada pregunta seleccionada nuevamente y se pedía a los entrevistados que indicaran si encontraban algún grado de dificultad o inconveniente. Para esto se utilizó el método de prueba derivado de la teoría cognitiva, acorde con lo planteado por Wild et al. (20), quienes consideran que se debe preguntar a la persona sobre el significado del ítem y solicitarle que lo "piense en voz alta" (think-aloud) reformulándolo con sus propias palabras para comprobar si se conservaba su significado (paraphrasing). En la fase de trabajo de campo se registró el tiempo transcurrido con el fin de poder determinar la viabilidad del cuestionario.

La aplicación de la versión adaptada del PCAT en las entrevistas cognitivas fue antecedido por la firma del consentimiento informado de cada uno de los participantes. Esta investigación no presentó riesgos para los seres humanos, al seguir las normas establecidas en la Resolución 08430 de 1993 de Colombia y la Declaración de Helsinki.

Para la ejecución del proyecto se contó con la aprobación y apoyo financiero de la Vicerrectoría de Investigaciones y Postgrados de la Universidad de Caldas, Colombia (Código del proyecto 0880113).

\section{Resultados}

\section{Valoración de la adecuación de versiones iberoamericanas en lengua española y adaptación al contexto colombiano}

En esta fase se consolidaron todos los resultados obtenidos con el grupo de expertos, separando los ítems que indagaban aspectos administrativos de los que evaluaban las funciones de la APS; por último, se contabilizaron todos los ítems calificados en cada uno de los niveles I, II, III y IV (Tabla 2).

Tabla 2. Resultados del proceso de adaptación por parte del grupo de expertos de los cuestionarios CO-PCAT-PE y CO-PCAT-FE

\begin{tabular}{|c|c|c|c|c|c|c|c|c|}
\hline \multirow{2}{*}{$\begin{array}{l}\text { Calificación } \\
\text { de los ítems }\end{array}$} & \multicolumn{4}{|c|}{ No. de ítems de las funciones esenciales y secundarias de APS } & \multicolumn{4}{|c|}{ No. de ítems complementarios* } \\
\hline & $\begin{array}{c}\text { PCAT -PE } \\
\text { primera revisión }\end{array}$ & $\begin{array}{l}\text { PCAT -PE } \\
\text { versión final }\end{array}$ & $\begin{array}{c}\text { PCAT-FE } \\
\text { primera revisión }\end{array}$ & $\begin{array}{c}\text { PCAT-FE } \\
\text { versión final }\end{array}$ & $\begin{array}{c}\text { PCAT -PE } \\
\text { primera revisión }\end{array}$ & $\begin{array}{l}\text { PCAT-PE } \\
\text { versión final }\end{array}$ & $\begin{array}{c}\text { PCAT-FE } \\
\text { primera revisión }\end{array}$ & $\begin{array}{c}\text { PCAT-FE } \\
\text { versión final }\end{array}$ \\
\hline Nivel I** & 89 & 89 & 69 & 69 & 47 & 46 & 58 & 57 \\
\hline Nivel II** & 65 & 65 & 91 & 91 & 37 & 38 & 48 & 49 \\
\hline Nivel III** & 2 & 2 & 1 & 1 & 6 & 6 & 6 & 6 \\
\hline Nivel IV** & 4 & 4 & 7 & 7 & 12 & 12 & 10 & 10 \\
\hline Total & 160 & 160 & 168 & 168 & 102 & 102 & 122 & 122 \\
\hline
\end{tabular}

* Se incluyeron encabezados, información introductoria o instrucciones para diligenciar el cuestionario, información general y la información para finalizar la entrevista.

** Nivel I (ítem equivalente, podría utilizarse el mismo enunciado), nivel II (cambios mínimos necesarios para su adaptación), nivel III (cambios mayores necesarios para su adaptación) y nivel IV (ítem no equivalente, debería excluirse).

Fuente: elaboración propia. 
ISSN-PRINT

1794-9831

E-ISSN 2322-7028

Vol. 15 No. 2

Jul - Dic 2018

Cúcuta, Colombia

\section{Revisión internacional por investigadores de la Colaboración Iberoamericana PCAT (IA-PCAT)}

Como resultado de esta fase, las investigadoras internacionales de Argentina, Uruguay y España, analizaron los ajustes propuestos en Colombia y a partir del trabajo realizado se redactó la versión inicial de los cuestionarios; a continuación se elaboró un listado de Ítems sobre los que se debía prestar mayor atención durante las pruebas preliminares, teniendo en cuenta los siguientes criterios: Ítems que sufrieron modificaciones importantes en las fases previas por haber sido valorados en el nivel III, y los que fueron propuestos para conformar la versión colombiana con una valoración de nivel II de adecuación.

\section{Pruebas preliminares: entrevistas cognitivas PCAT-PE y PCAT-FE}

Se realizaron 7 entrevistas a personal que trabaja en salud y que participa en la ejecución de la estrategia de APS (PCAT-PE) y 4 a gestores o directivos (PCATFE). Para la aplicación de las pruebas preliminares se tuvieron en cuenta las siguientes variables: edad, formación académica (Médicina, Enfermería, Psicólogía, Odontólogía, Trabajo social, Agente de salud pública, otro), trabajadores del sector de salud, rol (gestor o directivo, profesional o ejecución de la estrategia en APS) y ámbito de desempeño (nacional, departamental, municipal) (Tabla 3).

Tabla 3. Características de las personas participantes en las pruebas preliminares de la versión colombiana del CO-PCAT-PE y CO-PCAT-FE

\begin{tabular}{|c|c|c|}
\hline \multirow[b]{2}{*}{ Características de las personas participantes } & \multicolumn{2}{|c|}{ Versión del cuestionario } \\
\hline & PCAT-PE* & PCAT-FE+ \\
\hline Edad promedio en años (desviación estándar) [rango] & $\begin{array}{c}40,43(8,384)[27 \\
\text { años - } 53 \text { años }]\end{array}$ & $\begin{array}{c}43,00(10,985)[28 \\
\text { años - } 54 \text { años }]\end{array}$ \\
\hline \multicolumn{3}{|l|}{ Sexo } \\
\hline Mujer (n) & 6 & 4 \\
\hline Hombre (n) & 1 & 0 \\
\hline \multicolumn{3}{|l|}{ Formación académica } \\
\hline Enfermero & 2 & 2 \\
\hline Fisioterapeuta & 1 & 1 \\
\hline Fonoaudiólogo & 0 & 1 \\
\hline Agente de salud Pública & 1 & 0 \\
\hline Psicólogo & 1 & 0 \\
\hline Técnico en saneamiento ambiental & 1 & 0 \\
\hline Trabajador social & 1 & 0 \\
\hline \multicolumn{3}{|l|}{ Sector de salud en que se desempeña } \\
\hline Público & 7 & 4 \\
\hline \multicolumn{3}{|l|}{ Rol que desempeña } \\
\hline Gestor o directivo & 0 & 4 \\
\hline Profesional o persona que ejecuta la estrategia de APS & 6 & 0 \\
\hline Investigador en APS & 1 & 0 \\
\hline \multicolumn{3}{|l|}{ Ámbito de desempeño } \\
\hline Nacional & 0 & 0 \\
\hline Departamental & 1 & 0 \\
\hline Municipal & 6 & 4 \\
\hline
\end{tabular}

Durante la aplicación de las pruebas preliminares de ambos cuestionarios, se verificaron algunas preguntas que durante las fases de revisión por parte de los expertos y los investigadores de la Colaboración IAPCAT, requerían ser analizadas y determinar si se conservaban o no según su equivalencia semántica, práctica y cultural. A continuación se hace referencia a cada una:
- Proveedor de atención primaria: no tiene cambios porque las respuestas dadas por los entrevistados coinciden con la intencionalidad de la pregunta de referencia; uno de los términos utilizados por los encuestados para referirse a éste fue "entidad que proporciona la atención primaria de salud".

- Trabajo con agentes comunitarios de salud: los encuestados comprendieron el contenido del ítem; 
se pudo constatar cuando manifestaron que se refería por ejemplo a: "líder comunitario", "trabajo conjunto con autoridades locales", "agentes en salud".

- Medicina familiar: los entrevistados consideraron este término como adecuado para su aplicación en el contexto colombiano; algunos la denominaron como "medicina comunitaria", "salud familiar", "atención familiar".

- Doptone: tras la entrevista cognitiva, los encuestados comprendieron el contenido de la pregunta; para referirse a este término utilizaron otros como "estetoscopio", "equipo biomédico para escuchar la fetocardia", "monitor de ritmo cardíaco", "monitor fetal" y "doppler".

- Adicciones: al realizar la prueba cognitiva se encontró que los entrevistados asocian el término adicción a "apego incontrolado", "hábito de consumir drogas", "dependencia", "uso/abuso de sustancias psicoactivas", "necesidad de alguien o algo". Llamó la atención que ninguno de los profesionales, gestores o directivos incluyeran el juego o la ludopatía como adicción, solo mencionaron el consumo de sustancias psicoactivas.

- Formulación de anticoagulantes orales: al indagar sobre la formulación de este tipo de medicamentos, los entrevistados manifestaron que sí se formulan, por lo cual se constata la equivalencia práctica de este ítem al incluirse en los cuestionarios de aplicación al contexto colombiano.

En cuanto a la viabilidad del cuestionario, la administración del PCAT-PE requirió en promedio 99 minutos [mínimo: 60 minutos y máximo: 150 minutos] y el PCAT-FE necesitó en promedio 90 minutos [mínimo: 60 minutos y máximo 120 minutos]. En el cuestionario PCAT-PE predominaron las siguientes características: el grado de dificultad para responder las preguntas fue moderado (media=2,71). El grado de comprensión fue elevado y el contenido de las preguntas calificado como altamente interesante (media $=3,85$ y media $=3,71$ respectivamente). El lenguaje y la adecuación del trato recibieron una puntuación elevada (media $=3,14$ ). Quienesrespondieron el cuestionario PCAT-FEvaloraron el grado de dificultad para responder las preguntas como bajo (media=2), el grado de comprensión y el contenido elevado (media $=3,66$ y media $=3,33$ respectivamente), el lenguaje y el trato también elevados (media=3,33).
De manera particular, se hace énfasis en los siguientes ítems, los cuales requirieron modificaciones:

\section{PCAT-PE}

- “Aproximadamente qué porcentaje de sus usuarios tienen cobertura limitada o requiere autorización de los servicios?": se cambió el término "cobertura" por "plan de salud" y se incluyó el conector "y", quedando finalmente "¿Aproximadamente qué porcentaje de sus usuarios tienen un plan de salud limitado y/o que requiera autorización de las remisiones?"

- “QQué porcentaje de los usuarios de su institución cree usted que utiliza su consultorio para todas sus necesidades relacionadas con la salud y la enfermedad (sin contar urgencias o remisiones por servicios especializados)?": la palabra "Consultorio" presentó dudas, ya que en Colombia se utiliza consultorio más para el salón donde se realiza la consulta, se cambió por "institución", quedando "¿Qué porcentaje de los usuarios de su institución cree usted que utiliza su institución para todas sus necesidades relacionadas con la salud y la enfermedad (sin contar urgencias o remisiones por servicios especializados)?"

- “Cómo se resuelven las prestaciones que su servicio no ofrece? (Responda todas)": se dudó sobre la pertinencia del término "prestaciones" ya que una prestación en Colombia es una atención directa, en el contexto de la pregunta, por lo que el ítem se modificó y quedó "¿Cómo se resuelven las solicitudes de atención que su servicio no ofrece? (Responda todas)".

- "Cuando la institución de salud está cerrada, ¿usted tiene un número de teléfono al que los usuarios puedan llamarlo y conseguir ayuda si se enferman?": los participantes dudaron al responder la pregunta. Al respecto el ítem se reformuló así: Cuando su institución de salud está cerrada, ¿Los usuarios tienen el número de teléfono al que puedan llamar y conseguir ayuda si se enferman?

\section{PCAT-FE}

- ¿Cuál es el porcentaje aproximado de usuarios que atiende su institución de salud con cada una de las siguientes coberturas? Si no atiende ninguno de alguna modalidad, ponga cero": tras la entrevista, se
E-ISSN 2322-7028

Vol. 15 No. 2

Jul - Dic 2018

Cúcuta, Colombia 
ISSN-PRINT

1794-9831

E-ISSN 2322-7028

Vol. 15 No. 2

Jul - Dic 2018

Cúcuta, Colombia dudó si son "coberturas", por lo que el ítem quedó “¿Cuál es el porcentaje aproximado de usuarios que atiende su institución de salud con cada una de los siguientes tipos de aseguramiento en salud? Si no atiende ninguno de alguna modalidad, ponga cero."

- “Aproximadamente qué porcentaje de los usuarios de su institución de salud tienen una cobertura que limite o requiera aprobación de las remisiones?": La palabra "cobertura" tiene significado diferente en el contexto Colombiano ya que hace relación al cobrimiento de personas sobre el total de la población; por lo que el ítem se reformuló y quedó "¿Aproximadamente qué porcentaje de los usuarios de su institución de salud tiene un plan de salud que limite y/o requiera aprobación de las remisiones?"

- “En su institución de salud se pregunta a sus usuarios sobre sus opiniones o intenciones cuando se planifica su tratamiento o atención, o la de un miembro de su familia?": la palabra intención generó dudas, por lo que se reformuló el ítem así: ¿En su institución de salud se pregunta a sus usuarios sobre sus opiniones cuando se planifica su tratamiento o atención, o la de un miembro de su familia?

\section{Versión final de los cuestionarios PCAT-PE y PCAT- FE}

A partir del proceso de adaptación del PCAT$\mathrm{PE}$, se incluyeron en la versión colombiana 182 ítems distribuidos en los apartados que conforman el cuestionario así: información administrativa, información general, atributos esenciales y secundarios de la APS y otras características del servicio o institución de salud. En cuanto al proceso de adaptación del PCATFE, se incluyeron en la versión colombiana un total de 197 ítems distribuidos en los apartados que conforman el cuestionario. Particularmente, se incluyeron 144 ítems en los atributos tanto esenciales como secundarios de la APS (Tabla 4).

Tabla 4. Estructura del cuestionario PCAT-PE y PCAT-FE: versiones finales para Colombia

\begin{tabular}{|lcc|}
\hline Consolidación de número de ítems que integran PCAT & & \\
\hline Secciones del cuestionario / Versiones & $\begin{array}{c}\text { PCAT-PE } \\
\text { Colombia }\end{array}$ & $\begin{array}{c}\text { PCAT-FE } \\
\text { Colombia }\end{array}$ \\
\hline Información administrativa & 4 & 3 \\
\hline Información general & 26 & 33 \\
\hline Atributos esenciales & & \\
\hline C. Primer contacto - Accesibilidad & $10(+1)^{\mathrm{b}}$ & $10(+1)^{\mathrm{b}}$ \\
\hline D. Continuidad de la atención & 13 & $13(+1)^{\mathrm{b}}$ \\
\hline E. Coordinación & $7(+1)^{\mathrm{b}}$ & $9(+1)^{\mathrm{b}}$ \\
\hline F. Coordinación (sistema de información) & 9 & $9(+1)^{\mathrm{b}}$ \\
\hline G. Atención Integral (servicios disponibles) & $28(+4)^{\mathrm{b}}$ & $26(+4)^{\mathrm{b}}$ \\
\hline H. Atención Integral (servicios proporcionados) & 18 & 17 \\
\hline Atributos secundarios & & $14(+2)^{\mathrm{b}}$ \\
\hline I. Enfoque familiar & $24(+2)^{\mathrm{b}}$ & $24(+2)^{\mathrm{b}}$ \\
\hline J. Orientación comunitaria & 10 & 10 \\
\hline K. Competencia cultural & 9 & $15(+2)^{\mathrm{b}}$ \\
\hline Otras características del servicio o institución de salud & 182 & 197 \\
\hline Total & & \\
\hline
\end{tabular}

${ }^{b}$ ítem adicional incluido en la versión colombiana

Fuente: elaboración propia 


\section{Discusión}

La formación académica y experiencia de los expertos que participaron en la estrategia de APS, permitió establecer con elevado consenso las versiones de los cuestionarios PCAT-PE y PCAT-FE para el contexto colombiano.

Acorde con Shi et al. (3) este trabajo presenta un proceso de adaptación transcultural riguroso que supera a una simple traducción de los cuestionarios y proporciona información sobre la factibilidad de su aplicación como instrumentos de investigación en la región de Iberoamérica. Según Guillemin (21), Beaton et al. (18) y Berra et al. (22), el método de adaptación transcultural propuesto por diversos autores a nivel internacional consiste en llevar a cabo más fases que las realizadas aquí, porque incluyen traducción directa y retrotraducción. No obstante, para el desarrollo de este estudio se tuvieron en cuenta versiones previamente adaptadas mediante ese procedimiento en países de habla hispana como Argentina, Uruguay y España, lo que facilitó que a partir de esas versiones en Castellano se omitiera la traducción-retrotraducción y así se consiguió una mejor equivalencia mediante un proceso más eficiente, con la garantía que ofreció la revisión internacional de personas de la Colaboración IAPCAT, que habían participado en los estudios previos.

En Argentina dado que el sistema de salud es diferente, derivado del proceso de adaptación transcultural, ítems relacionados con las características de los centros, los proveedores, el equipo de salud y la cobertura de la atención en salud, se sometieron a cambios importantes, según lo reportado por Berra et al. en dos publicaciones $(6,22)$. En el contexto colombiano, de igual manera, se requirieron cambios en algunos ítems de los cuestionarios, tanto en la versión PCAT-PE como en la versión PCAT-FE.

Este cuestionario ha sido adaptado en otros países encontrando que su validez y fiabilidad fueron adecuadas y similares a la versión original, como el caso de Brasil (9, 23, 24), Argentina (6), España (13) y Uruguay (11), de ahí que pueden ser utilizados para la identificación, seguimiento y evaluación de las funciones de la APS en los servicios de salud, así como en estudios comparativos de un país o entre países (25-27). De igual forma en España, se evaluó la validez y confiabilidad de los cuestionarios encontrando que son adecuadas (14) y en Uruguay también se realizó el proceso de validación del PCAT-PE (11).

Pasarín et al. (13) ha descrito que este tipo de estudios permite comparaciones nacionales e internacionales, partiendo de criterios unificados y/o armonizados para la evaluación de la APS.

El desarrollo de este trabajo permite resaltar las ventajas de llevar a cabo procesos colaborativos y de trabajo en red con un objetivo común que, para este caso, ha sido la búsqueda de la armonización a nivel iberoamericano del conjunto de cuestionarios PCAT. En este mismo sentido, la definición del trabajo en red propuesto por Albornoz y Alfaraz C (28), abarca un conjunto de "mecanismos estratégicos para promover el intercambio de información, experiencias, conocimiento y contribuir con la cooperación técnica en el nivel nacional e internacional en diferentes áreas temáticas". Según Puente (29) y Stange et al. (30) el trabajo colaborativo permite una mejor comprensión de las necesidades y agendas (políticas), la construcción de esfuerzos conjuntos, la generación de nuevas ideas, que contribuyen con la toma de decisiones y sirve para unir gobierno, sector privado, sociedad civil, organizaciones no gubernamentales (ONG) y organizaciones internacionales, promoviendo la formación de alianzas. Finalmente como lo destaca Bresick G et al., del desarrollo de los procesos de adaptación transcultural se derivan recomendaciones, como la necesidad de fortalecer las mejores prácticas regionales y nacionales en la APS y la formulación de políticas de salud que involucren la capacitación de profesionales y gerentes sobre este tema (31, 32).

\section{Conclusiones}

- Se realizó la traducción de las versiones extensas de los cuestionarios PCAT-PE y PCAT-FE; fue evaluada su adecuación al sistema de salud Colombiano obteniendo las versiones de los cuestionarios CO-PCAT-PE y CO-PCAT-FE de APS, con equivalencia semántica, práctica y cultural a las originales, 
garantizando su validez de contenido a través de un proceso colaborativo internacional de adaptación transcultural.

- Los cuestionarios permitirán valorar el grado en que la APS cumple sus funciones esenciales y secundarias desde la perspectiva de profesionales y gestores o directivos que implementan la estrategia, lo cual facilitará, en el mediano plazo, realizar comparaciones nacionales e internacionales.

- Adicionalmente, derivado de este trabajo, se confirma la utilidad de la propuesta de adaptación transcultural del PCAT en los países de habla hispana realizada por la Colaboración IA-PCAT, con la modificación de algunas fases respecto de la metodología habitual.

\section{Agradecimientos}

Las autoras agradecen a los expertos nacionales Claudia Piedad Estrada Rueda, Norman Giovanni Apraez Ippolito, Ivanoba Pardo Herrera, Juan Eduardo Guerrero Espinel y Nancy Ramírez Olarte; al Programa de Jóvenes Investigadores e Innovadores de Colciencias; a la Universidad de Caldas y al Grupo de Investigación Promoción de la Salud y Prevención de la Enfermedad, por la oportunidad brindada para el desarrollo de este proyecto.

\section{Conflicto de Intereses}

Los autores declaran no tener ningún conflicto de intereses.

\section{Referencias bibliográficas}

1. Sperber AD. Translation and validation of study instruments for cross-cultural research. Gastroenterology. 2004; 126(1 Suppl 1):S124-8.

2. Prates ML, Machado JC, Silva LSD, Avelar PS, Prates LL, et al. Performance of primary health care according to PCATool instrument: a systematic review. Ciênc. saúde colet. 2017 22(6):1881-1893.

3. Shi L, Pinto Masís D, Guanais FC. Measurement of Primary Care: Report on the Johns Hopkins Primary Care Assessment Tool. 2012 Guidance and Recommendations. Contract No.: IDB-TN-482.

4. Vélez Álvarez C, Giraldo Osorio A. La atención primaria de salud desde la perspectiva del equipo de salud en una institución de primer nivel de atención. Rev cienc cuidad. 2015; 10(2):76-84.

5. Starfield B, Shi L, Macinko J. Contribution of primary care to health systems and health. Milbank Memorial Fund. 2005; 83(3):457-5.

6. Berra S, Audisio Y, Mántaras J, Nicora V, Mamondi V, Starfield B. Adaptación cultural y al sistema de salud argentino del conjunto de instrumentos para la evaluación de la Atención Primaria de la Salud Rev Argent Salud Pública. 2011; 2(8):6-14.

7. De Oliveira MMC, Harzheim E, Riboldi J, Duncan BB. PCATool-Adulto-Brasil: uma versão reduzida. Rev Bras Med Fam Comunidade. 2013; 8(29):256-63.

8. Harzheim E, De Oliveira MMC, Agostinho MR, Hauser L, Stein AT, Gonçalves MR, et al. Validação do instrumento de avaliação da atenção primária à saúde: PCATool-Brasil adultos. Rev Bras Med Fam Comunidade. 2013; 8(29):274-84.

9. Hauser L, De Castro RCL, Vigo Á, Da Trindade TG, Gonçalves MR, Stein AT, et al. Tradução, adaptação, validade e medidas de fidedignidade do Instrumento de Avaliação da Atenção Primária à Saúde (PCATool) no Brasil: versão profissionais de saúde. Revista Brasileira de Medicina de Família e Comunidade. 2013; 8(29):244-55.

10. Harzheim E, Starfield B, Rajmil L, Álvarez-Dardet C, Stein AT. Consistência interna e confiabilidade da versão em português do Instrumento de Avaliação da Atenção Primária (PCATool-Brasil) para serviços de saúde infantil. Cad Saúde Pública. 2006; 22:1649-59.

11. Pizzanelli M, Ponzo J, Buglioli M, Toledo A, Casinelli M, Gómez A. Validación de Primary Care Assessment Tool (PCAT) en Uruguay. Rev Med Urug. 2011; 27(3):187-9. 
12. Berra S, Rocha KB, Rodríguez Sanz M, Pasarín MI, Rajmil L, et al. Properties of a short questionnaire for assessing Primary Care experiences for children in a population survey. BMC Public Health. 2011; 11(285).

13. Pasarín MI, Berra S, González A, Segura A, Tebé C, et al. Evaluation of primary care: The "Primary Care Assessment Tools-Facility version" for the Spanish health system. Gac Sanit. 2013; 27(1):12-8.

14. Rocha K, Rodríguez-Sanz M, Pasarín M, Berra S, Gotsens M, Borrell C. Assessment of primary care in health surveys: a population perspective. Eur J Public Health. 2011; 21(2):1-5.

15. HX-Wang H, CS-Wong M, YS-Wong S, Ling-Tang J, Wang-Guangzhou JJ, Li-Bauhinia DK, et al., editors. Adaption of Primary Care Assessment Tool for Evaluation of Primary Care Achievements Under China>s Healthcare Reform. 13 Congress on Public Health; 2012; Addis Adaba, Ethiopia2012.

16. Lee H, Choi Y, Sung N, Kim S, Chung S, et al. Development of the Korean primary care assessment tool-measuring user experience: tests of data quality and measurement performance. Int $\mathrm{J}$ Qual Health Care. 2009; 21(2):103-8.

17. Centro de Investigación Epidemiológica y en Servicios de Salud. Colaboración IA-PCAT Universidad Nacional de Córdoba: Berra Silvina; 2017

18. Beaton DE, Bombardier C, Guillemin F, Bosi-Ferraz M. Guidelines for the Process of Cross-Cultural Adaptation of Self-Report Measures. Spine. 2000; 25(24):3186-91.

19. Guillemin F, Bombardier C, Beaton D. Cross-cultural adaptation of health-related quality of life measures: literature review and proposed guidelines. Journal of clinical epidemiology. 1993; 46(12):1417-32.

20. Willis GB, Statistics NCfH. Cognitive interviewing and questionnaire design: A training manual: US Department of Health and Human Services, Centers for Disease Control and Prevention, National Center for Health Statistics; 1994.

21. Guillemin F. Cross-cultural Adaptation and Validation of Heatth Status Measures. Scandinavian Journal of Rheumatology. 1995; 24(2):61-3.

22. Berra S, Hauser L, Audisio Y, Mántaras J, Nicora V, De-Oliveira MMC, et al. Validez y fiabilidad de la versión argentina del PCAT-AE para evaluar la atención primaria de salud. Rev Panam Salud Publica. 2013; 33(1):30-9.

23. Pereira D'Avila O, Da Silva Pinto LF, Hauser L, Rodrigues Gonçalves M, Harzheim E. The use of the Primary Care Assessment Tool (PCAT): an integrative review and proposed update. Ciênc. saúde colet. 2017; 22(3):855-65.

24. Pinto LF, Harzheim E, Hauser L, D'Avila OP, Gonçalves MR, Travassos P, et al. A qualidade da Atenção Primária à Saúde na Rocinha-Rio de Janeiro, Brasil, na perspectiva dos cuidadores de crianças e dos usuários adultos. Ciênc. saúde colet. 2017; 22:771-81.

25. Mei J, Liang Y, Shi L, Zhao J, Wang Y, Kuang L. The development and validation of a rapid assessment tool of Primary Care in China. BioMed Research International; 2016.

26. Macinko J, Jimenez G, Cruz Peñate M. Primary care performance in Dominica. Rev Panam Salud Publica. 2015; 37(2):104-12.

27. Harzheim E, Pinto LF, Hauser L, Soranz D. Avaliação dos usuários crianças e adultos quanto ao grau de orientação para Atenção Primária à Saúde na cidade do Rio de Janeiro, Brasil. Ciênc. saúde colet. 2016; 21:1399-408.

28. Albornoz ME, Alfaraz C. Redes de conocimiento: construcción, dinámica y gestión. Buenos Aires: Redes, Centro de Estudios sobre Ciencia, Desarrollo y Educación Superior 2006.

29. Puente Arnao G. Gestión de redes en la OPS/OMS Brasil: conceptos, prácticas y lecciones aprendidas. Gestión de redes en la OPS/OMS Brasil: conceptos, prácticas y lecciones aprendidas: Organización Panamericana de la Salud; 2008. 
ISSN-PRINT

$1794-9831$

E-ISSN 2322-7028

Vol. 15 No. 2

Jul - Dic 2018

Cúcuta, Colombia
30. Stange KC, Etz RS, Gullett H, Sweeney SA, Miller WL, et al. Metrics for assessing improvements in primary health care. Annual review of public health. 2014;35:423-42.

31. Bresick G, Sayed AR, Le Grange C, Bhagwan S, Manga N, Hellenberg D. Western Cape Primary Care Assessment Tool (PCAT) study: Measuring primary care organisation and performance in the Western Cape Province, South Africa (2013). Afr J Prim Health Care Fam Med. 2016; 8(1):1-12.

32. Bresick G, Sayed AR, le Grange C, Bhagwan S, Manga N. Adaptation and cross-cultural validation of the United States Primary Care Assessment Tool (expanded version) for use in South Africa. Afr J Prim Health Care Fam Med. 2015; 7(1):1-11. 